\title{
A Review of the Research on the Influence of Cavitation and Abrasion on Hydroturbine Performance
}

\author{
Yu Tianchen ${ }^{1, a^{*}}$ \\ ${ }^{1}$ Hohai University College of Hydrology and Water Resources, Gulou, Nanjing, Jiangsu, China
}

\begin{abstract}
Large and medium sized water conservancy projects generally have comprehensive benefits such as flood control, water supply, irrigation and power generation. As the main hydraulic power generation equipment, the safe and economic operation of hydroturbine generator sets is of vital importance. For a hydroturbine affected by the flow of water in the running process, the paper explores the cavitation and abrasion phenomena, analyses the phenomenon of resource waste, energy waste and material waste caused by unreasonable protection of water turbine, and introduces relevant protective measures. These results further enhance the understanding of the corrosion resistance of the hydroturbine and could be used to optimize the hydroturbine protection techniques.
\end{abstract}

\section{Introduction}

A turbine is a power machine that converts the energy of water flow into rotational mechanical energy. Modern water turbines are mostly installed in hydropower stations to drive generators to generate electricity. In the process of generating electricity, the water in the upstream reservoir is directed to the turbine by the water pipe, which drives the turbine runner to rotate, which in turn drives the generator to generate electricity. In 2010, the installed capacity of hydropower in China reached 211 million $\mathrm{kW}$, and it is expected to reach 300 million in 2020. The rapid development of turbine technology in China has generated many economic benefits and strategies concerning energy conservation, emission reduction, environmental pollution reduction, creating positive conditions for China's economic and social development.

As the sediment concentration in most of the rivers of China is high, the water contains sediment grain of different size and this can cause losses to the turbine ${ }^{[1]}$, if we don't take timely protective measures. Furthermore, unexpected working conditions can reduce the safe and stable operation of the turbine, causing serious influence the normal operation of hydropower station, such as fatigue $^{[2]}$ and instability ${ }^{[3]}$.

Water flow on the turbine can cause cavitation erosion, hydraulic erosion and sediment caused by abrasion, wear phenomenon. Data in 2002 showed that there were 132 sets of sediment erosion on hydroturbines of large and medium-sized hydropower stations in China, with a total power capacity of over 12 million $\mathrm{kW}$ (excluding the Three Gorges). About $30 \%$ of these ( 6.6 million $\mathrm{kW}$ ) were small and medium-sized hydropower stations, with a total of 13,000 units ${ }^{[4]}$. By the end of 2013, China's total installed capacity of hydraulic power generation exceeded
280 million $\mathrm{kW}$. However, the wear of turbine overflow components every year led to the replacement and repair of power generation equipment, which resulted in huge expenditure and obvious damage to the benefits of the power station $^{[5]}$.

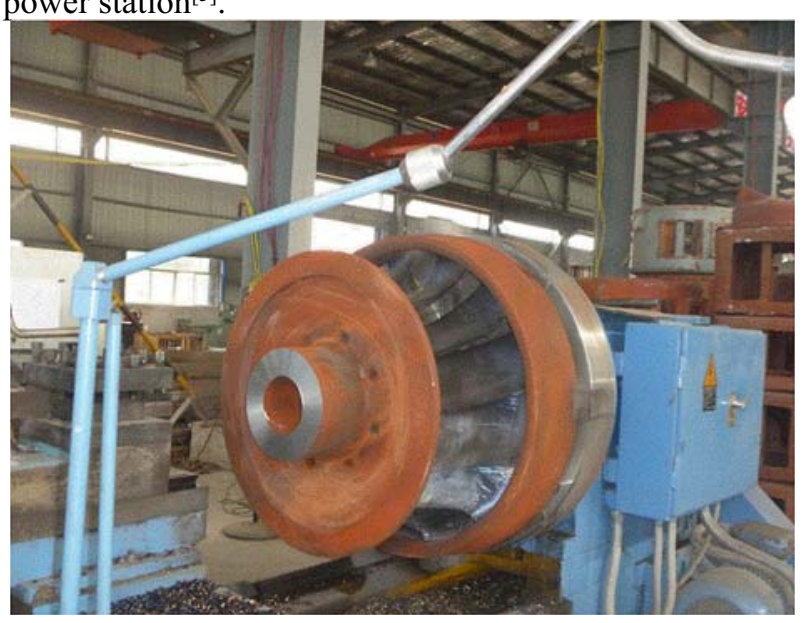

Figure1 Hydroturbine maintenance

The erosion of water flow has greatly increased the cost of maintenance and reconstruction of turbine equipment, and needs serious attention in the design of water conservancy projects, equipment material selection and material protection. For this reason, authors such as Chunxia Yang et al. ${ }^{[6]}$ have studied the wear-erosion mechanism in rotating surfaces, providing in-depth information of surface wear and morphology.

\footnotetext{
* Corresponding author: ${ }^{\mathrm{a}} 1946219468 @ q q . c o m$
} 


\section{Research Progress on Cavitation and Abrasion of Hydraulic Turbines}

\subsection{Current Situation and Protection of Cavitation Erosion on Hydraulic Turbines}

Cavitation is a widely existing corrosion mode, which is related to heterogeneous media such as air, steam and micro-particles in water, as well as the temperature and pressure of water. The cavitation erosion phenomenon of water turbine is mainly manifested in the continuous flow of liquid flow on the edge wall of the material. First, the material is deformed, and then falls off, through the mechanical impact, chemical, electrochemical action, destroying the surface of the flow, and changing the mechanical energy characteristics. It produces noises and has a negative impact on the mechanical performance.

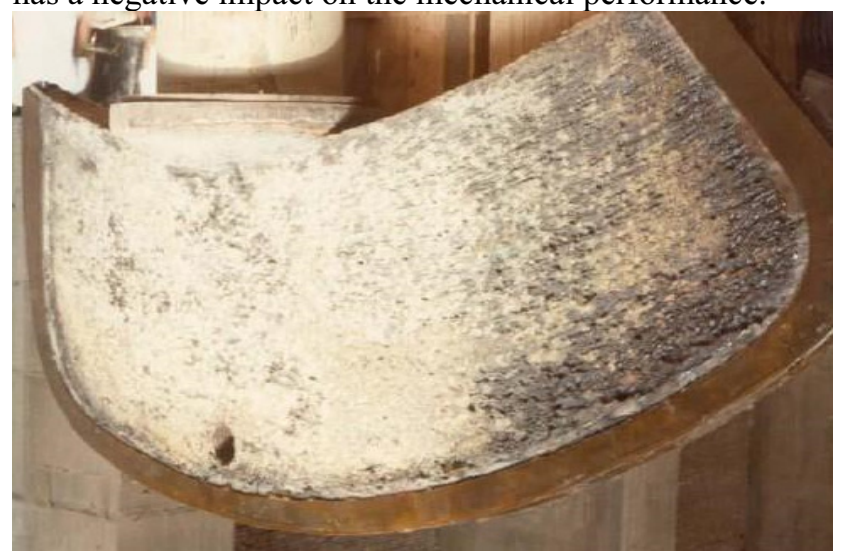

Figure2 Cavitation of hydraulic turbine

Turbine hollow corrosion phenomenon is reflected in the turbine flow passage, as the low pressure area formed here collapse into the downstream high pressure area, leading to a series of effects on the flow surface. The main types are airfoil, clearance, cavity and local cavitation. Cavitation in a turbine will produce a series of noises, affect the efficiency and output of the turbine, shorten the service life of the turbine. In the operation of a turbine, because the flow velocity at the outlet of the blade and the casing is relatively large, the pressure decreases with the increase of the flow velocity and tends to produce local vacuum phenomenon. At the same time, it is accompanied by a strong vibration wave, impacting equipment and components, and resulting in local parts damaged by steam erosion. Cavitation occurs in the operation of the turbine and may be caused by improper design and manufacturing, such as improper blade profile, material selection, etc. On the other hand, the operating conditions may deviate from the design requirements for a long time, such as low head, load and suction height, which may cause cavitation in long-term operation.

At present, the main measures for the prevention of cavitation erosion in engineering are as follows: in the design selection and installation of the turbine, the appropriate cavitation coefficient is selected; in the design of the choice of good airfoil, the best material is used; in terms of engineering measures, low water head and low output are avoided, and attention is paid to sand control and discharge; in the process, the air feeder is used to destroy the vortex zone with high vacuum. In terms of operation, appropriate operation area, timely maintenance, surface coating are implemented, and when the equipment shows local cavitation, a timely repair or replacement is performed, to ensure the safe operation of the unit.

\subsection{Current Situation and Protection of Abrasion on Hydraulic Turbines}

When the turbine is working, if the water flow through it contains a lot of sand and mud, then the hard sand particles will impact and wear the surface of flowing parts, so that the machine parts fatigue or even damage, that is, the turbine shows wear mechanisms. Turbine wear will have serious consequences, with at least a maintenance treatment, or in worse cases, a replacement of parts, such as the wheel. Turbine wear will also aggravate cavitation damage, and increase the vibration of turbine. When the water guide mechanism of the turbine wears seriously, the water leakage will increase, which will affect the normal stop. If the turbine phases the regulation operation, it will increase the power loss of phase regulation, or even fail under this mode. The failure intensity of sand and mud wear is related to the characteristics of sand flow, the material characteristics of turbine flow components, the working and operating conditions of the turbine. When water flow containing sand with high velocity impinges on solid wall surface, sometimes the stress generated by one impact may exceed the yield limit of the material and lead to plastic deformation of the material. Even a small impact stress will cause fatigue failure of the material due to frequent action. Sometimes sand wear and cavitation occur simultaneously, resulting in a more complex combined failure process.

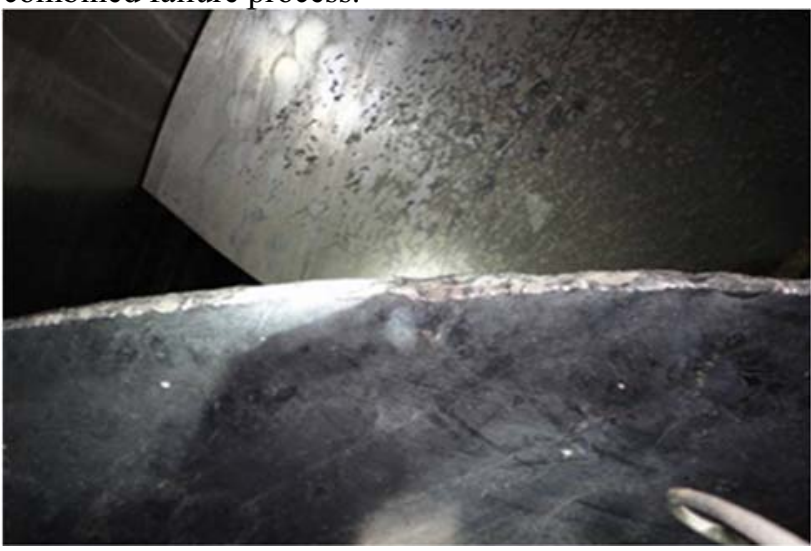

Figure3 Abrasion of hydraulic turbine

In recent years, a large number of laboratory and field experiments have been carried out on the mechanism of sand wear and various technical measures have been developed to prevent sand wear in hydraulic turbines. Research methods such as using guye two-phase flow model analyze the sediment distribution in the turbine; Therefore, the wear conditions of material surface under different particle size, impact Angle and flow velocity can be compared. Jet wear device is used to compare the wear 
degree of coating with different materials. The flow law of guide vane outlet and runner inlet of axial flow turbine in sand water can be simulated by establishing and transforming the device ${ }^{[7]}$, etc.

The existing results show that the effective methods for the mitigation and prevention of turbine abrasion are as follows:

The first is to adopt the sand blocking measure of hydraulic construction. The most fundamental measure to prevent the turbine from sand wear is to intercept the sand and prevent it from entering the turbine runner. Power stations should be built on the muddy sand river, and the water intake hub should consider preventing the sand and mud from entering the turbine as far as possible. Water conservancy projects are often equivalent to the reservoir sand pool, since it can deposit the sand in the reservoir area and reduce the amount of sand in the turbine working flow, especially intercepting the coarse sand into the turbine flow channel. The turbine sand content and water inlet and exhaust position of sand are known to be related ${ }^{[8]}$.

The second is to rationally choose the type and working parameters of water turbine. This is done by weighting the various factors from various aspects, carrying on comprehensive technical and economic comparison, referring to the adaptability of different types of turbine to different water flow conditions and treatment basis, and selecting the appropriate turbine form and working parameters. For the high head hydropower station on the muddy and sandy river, the flow velocity in the overflow element of the turbine is generally higher, which will cause serious wear. Under such conditions, the impingement type and mixed-flow turbine with higher anti-erosion performance are usually selected. Among them, the mixed-flow turbine has a longer working life and less power loss, and the impact turbine is more convenient for maintenance.

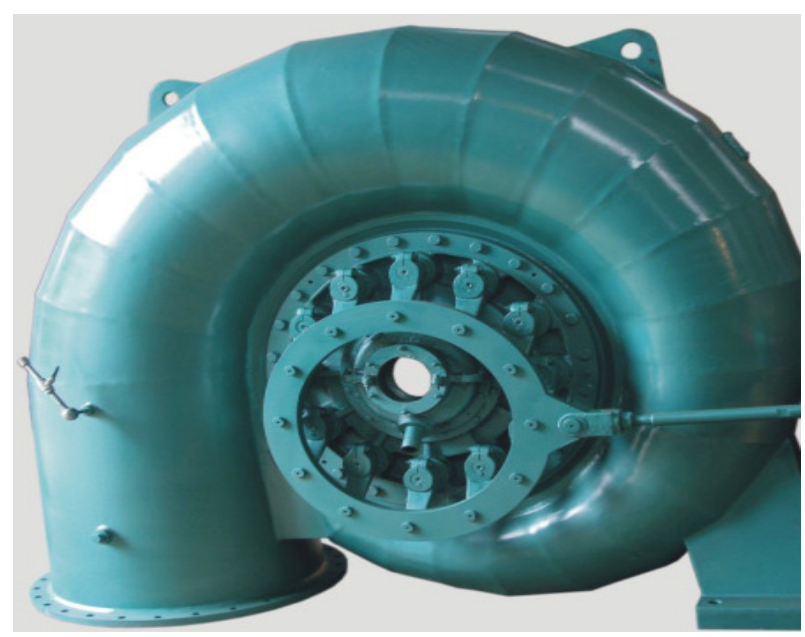

Figure4 Axial flow turbine

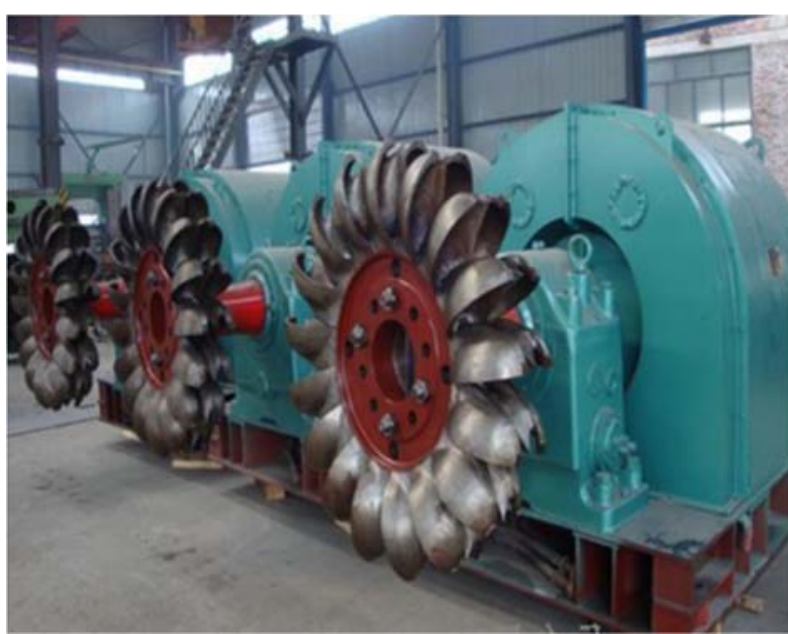

Figure5 Mixed-flow turbine

The third is to reasonably control the operation condition of the turbine suffering from sand and mud wear. Under severe sediment wear, the operation of turbine units often deviates from the optimal condition, and the average relative velocity of flow passage may be significantly reduced compared with the optimal efficiency condition. Therefore, according to the wear condition, the operation condition arrangement of the turbine can be improved appropriately, and the maintenance cycle can be extended, so as to ensure the normal operation of the turbine.

The fourth is to adopt the material with high anti-sand wear stability and wear-resisting protection technology. This includes the application of turbine wear design measures and the use of anti-wear material coating and special polymer materials of high strength and toughness, as to solve the high speed sand flow impact wear, improve the anti-wear conditions of turbine components, and protect hydraulic machinery parts easy to wear, in order to extend its service life and maintain a high efficiency operation. Typical materials are shown in Table $1^{[9]}$.

Table1 The materials to prevents cavitation and abrasion of a

\begin{tabular}{|c|c|}
\hline Target & Materials \\
\hline Prevent & ZG20SiMn \\
cavitation & 0Cr13Ni4Mo \\
& 0Cr13Ni5Mo \\
& 0Cr13Ni6Mo \\
& 0Cr16Ni5Mo \\
& 0Cr13Ni4CuMo \\
\hline Prevent & Epoxy emery \\
abrasion & SPH \\
& FM Anti-corrosion and wear \\
& resistant Powder layer \\
& GBI electrode surfacing layer \\
\hline
\end{tabular}




\section{Conclusions}

There are many sediment rivers in China and these can negatively affect the hydroturbines operation, together with cavitation and abrasion phenomena. At present, according to the existing conditions in China, the protection measures of hydraulic turbine mainly include the optimization of the layout of the hub and selection of the appropriate type to control the working condition reasonably. As result, these can prevent damage and control the hydro characteristics, such as reducing the flow velocity and sediment concentration, and promoting the impact resistance and wear resistance of the material.

China's hydroturbine technology has been at the forefront of the world, and its development is closely related to its energy demand. As important hydropower equipment, the hydroturbine is an indispensable core component of the hydropower industry. Its safe and stable operation are of great value to realize the economic and social benefits of water conservancy projects. Through the study of this paper, some basis is provided for the rational use and optimization of the protection measures of hydroturbines.

\section{References}

1. Peiyuan, Y., Zhifu, H. (2017) Damage and Protection Measures of Sediment Abrasion in Hydraulic Turbines. J. Jilin Water Conservancy, 60-62.

2. Xiaocui, C., Yuan, Z. (2020) Fatigue Life Study of Francis Pump Under Reverse Generation Conditions Based on Fluid Solid Coupling. J. Water 2020, 12: 1162.

3. Chengyi, L. (2020) Stability Optimization and Analysis of Bi-directional Shaft Extension Pump.

4. Zhigao, W. (2002) The Current Situation and the Progress of the Protection Measures of the Water Machine Abrasion in China. J. Water Conservancy and Hydropower Engineering Design, 1-4.

5. Yun, C., Xin, Z., Qingfeng, F. (2015) Research Status of surface Abrasion Resistance Technology of Hydraulic Turbine Blade. J. Jiangxi Science, 593-597.

6. Chunxia, Y., ShanShan, H., et al. (2020) MultiComponent Water Effects on Rotating Machines Disk Erosion. J. Water 2020, 12: 757.

7. Siyang, L., Shuai, Y., Yongzhong, Z., et al. (2019) Experimental Study on Sediment Wear of Moving Guide Vane of Water Turbine. J. China Rural Water Conservancy and Hydropower, 192-196.

8. Chaoyang, L. (1991) Introduction to Power Plants. Water Resources and Electric Power Press, Beijing.

9. Jianjun, X., Jianming, L. (2007) Progress in Research, Protection and Control Technology of Turbine Abrasion in China. J. China Rural Water Conservancy and Hydropower, 137-139. 